\title{
Correction: Global and regional estimates of cancer mortality and incidence by site: II. results for the global burden of disease $\mathbf{2 0 0 0}$ Kenji Shibuya*1, Colin D Mathers¹, Cynthia Boschi-Pinto², Alan D Lopez ${ }^{3}$ and Christopher JL Murray ${ }^{4}$
}

Address: ${ }^{1}$ Global Program on Evidence for Health Policy, World Health Organization, Geneva, Switzerland, ${ }^{2}$ Family and Community Health/ Child and Adolescent Health and Development, World Health Organization, Geneva, Switzerland, ${ }^{3}$ School of Population Health, University of Queensland, Herston, Australia and ${ }^{4}$ Executive Director, Evidence and Information for Policy, World Health Organization, Geneva, Switzerland

Email: Kenji Shibuya* - shibuyak@who.int; Colin D Mathers - mathersc@who.int; Cynthia Boschi-Pinto - pintoc@who.int; Alan D Lopez - A.Lopez@sph.uq.edu.au; Christopher JL Murray - murrayc@who.int

* Corresponding author

Published: 23 July 2003

BMC Cancer 2003, 3:20
Received: 17 July 2003

Accepted: 23 July 2003

This article is available from: http://www.biomedcentral.com/I47/-2407/3/20

(C) 2003 Shibuya et al; licensee BioMed Central Ltd. This is an Open Access article: verbatim copying and redistribution of this article are permitted in all media for any purpose, provided this notice is preserved along with the article's original URL.

After the publication of this work [1], we noticed the typographical errors in Tables 8, 9, 16, and 17: there were inconsistencies between the ranking of cancer mortality and incidence and their corresponding figures. Here we briefly present the results along with the revisions of the relevant tables since the ranking was corrected while the figures remained the same as the original.

Tables 1 and 2 represent the ranking of the number of deaths by cancer site in the world and three selected sub regions: African Region with a high child and adult mortality (AfrE), European Region with a very low child and adult mortality (EurA), and South East Asia Region with a low child and adult mortality (SearB). Lung cancer was the leading cause of cancer deaths in the world, accounting for $17 \%$ of total cancer mortality, followed by cancers of stomach (12\% of total), colon and rectum $(9 \%)$, liver $(9 \%)$, and breast $(7 \%)$. In males, lung, stomach, and liver cancers were the three most common causes of cancer deaths. The leading cause of cancer deaths among females was breast cancer, but lung cancer was already one of the largest causes of female cancer mortality.

The distribution of common cancer incidence was somewhat similar to that of mortality: lung cancer was the most common cancer in the world in 2000 , accounting for $13 \%$ of total cancer incidence, followed by cancers of colon and rectum ( $10 \%$ of total), breast (10\%), stomach (10\%), and liver (6\%) (Table 3). As in mortality distribution, there is a significant variation in the distribution of sitespecific cancer incidence by region (Table 4 ). The variations in the distribution of site-specific new cases of cancer by region were also similar to those observed in mortality distribution.

We regret any inconvenience that this inaccuracy in the tables might have caused. We wish to thank Dr. Robert Dubrow of Yale University School of Medicine for bringing this matter to our attention.

\section{References}

I. Shibuya K, Mathers CD, Boschi-Pinto C, Lopez AD and Murray CJL: Global and regional estimates of cancer mortality and incidence by site: II. results for the global burden of disease $\mathbf{2 0 0 0}$ BMC Cancer 2002, 2:37.

\section{Pre-publication history}

The pre-publication history for this paper can be accessed here:

http://www.biomedcentral.com/1471-2407/3/20/prepub 
Table I: Ranking of the global cancer deaths by site, 2000

\begin{tabular}{|c|c|c|}
\hline Site & Number of deaths (000s) & Proporion of total (\%) \\
\hline \multicolumn{3}{|l|}{ Both sexes } \\
\hline Trachea, bronchus, and lung & $1,201.1$ & 17.1 \\
\hline Stomach & 836.2 & 11.9 \\
\hline Colon and rectum & 607.3 & 8.7 \\
\hline Liver & 605.9 & 8.6 \\
\hline Breast & 468.9 & 6.7 \\
\hline Oesophagus & 431.2 & 6.1 \\
\hline Lymphomas and multiple myeloma & 332.3 & 4.7 \\
\hline Mouth and oropharynx & 319.6 & 4.6 \\
\hline Prostate & 263.6 & 3.8 \\
\hline Leukaemia & 260.1 & 3.7 \\
\hline Cervix uteri & 259.6 & 3.7 \\
\hline Pancreas & 222.6 & 3.2 \\
\hline Bladder & 177.4 & 2.5 \\
\hline Ovary & 128.6 & 1.8 \\
\hline Corpus uteri & 72.9 & 1.0 \\
\hline Melanoma of the skin & 65.1 & 0.9 \\
\hline \multicolumn{3}{|l|}{ Males } \\
\hline Trachea, bronchus, and lung & 877.3 & 22.5 \\
\hline Stomach & 512.0 & 13.2 \\
\hline Liver & 414.9 & 10.7 \\
\hline Colon and rectum & 311.8 & 8.0 \\
\hline Oesophagus & 273.6 & 7.0 \\
\hline Prostate & 263.6 & 6.8 \\
\hline Mouth and oropharynx & 222.4 & 5.7 \\
\hline Lymphomas and multiple myeloma & 168.6 & 4.3 \\
\hline Leukaemia & 145.3 & 3.7 \\
\hline Bladder & 123.9 & 3.2 \\
\hline Pancreas & 117.0 & 3.0 \\
\hline Melanoma of the skin & 35.1 & 0.9 \\
\hline \multicolumn{3}{|l|}{ Females } \\
\hline Breast & 466.3 & 14.9 \\
\hline Stomach & 324.2 & 10.4 \\
\hline Trachea, bronchus, and lung & 323.8 & 10.4 \\
\hline Colon and rectum & 295.5 & 9.4 \\
\hline Cervix uteri & 259.6 & 8.3 \\
\hline Liver & 191.0 & 6.1 \\
\hline Lymphomas and multiple myeloma & 163.7 & 5.2 \\
\hline Oesophagus & 157.6 & 5.0 \\
\hline Ovary & 128.6 & 4. 1 \\
\hline Leukaemia & 114.7 & 3.7 \\
\hline Pancreas & 105.6 & 3.4 \\
\hline Mouth and oropharynx & 97.2 & 3.1 \\
\hline Corpus uteri & 72.9 & 2.3 \\
\hline Bladder & 53.5 & 1.7 \\
\hline Melanoma of the skin & 30.1 & 1.0 \\
\hline
\end{tabular}


Table 2: Ranking of selected regional cancer deaths by site, 2000

\begin{tabular}{|c|c|c|c|c|c|c|c|c|}
\hline \multicolumn{9}{|c|}{ WHO sub region } \\
\hline \multicolumn{3}{|c|}{ AfrE (high child and adult mortality) } & \multicolumn{3}{|c|}{ EurA (very low child and adult mortality) } & \multicolumn{3}{|c|}{ SearB (low child and adult mortality) } \\
\hline Site & $\begin{array}{l}\text { Number } \\
\text { of deaths } \\
(000 s)\end{array}$ & $\begin{array}{l}\text { Proportion } \\
\text { of total (\%) }\end{array}$ & & $\begin{array}{l}\text { Number } \\
\text { of deaths } \\
(000 \mathrm{~s})\end{array}$ & $\begin{array}{l}\text { Proportion } \\
\text { of total (\%) }\end{array}$ & & $\begin{array}{l}\text { Number } \\
\text { of deaths } \\
(000 \mathrm{~s})\end{array}$ & $\begin{array}{l}\text { Proportion } \\
\text { of total (\%) }\end{array}$ \\
\hline \multicolumn{9}{|l|}{ Both sexes } \\
\hline Cervix uteri & 36.8 & 12.4 & $\begin{array}{l}\text { Trachea, bronchus, } \\
\text { and lung }\end{array}$ & 207.2 & 19.5 & $\begin{array}{l}\text { Trachea, bronchus, } \\
\text { and lung }\end{array}$ & 48.3 & 16.6 \\
\hline Liver & 33.3 & 11.2 & Colon and rectum & $14 \mid .8$ & 13.3 & Liver & 36.5 & 12.5 \\
\hline Breast & 23.0 & 7.8 & Breast & 92.7 & 8.7 & Breast & 29.1 & 10.0 \\
\hline $\begin{array}{l}\text { Mouth and } \\
\text { oropharynx }\end{array}$ & 21.4 & 7.2 & Prostate & 70.2 & 6.6 & Colon and rectum & 27.6 & 9.5 \\
\hline Oesophagus & 20.6 & 7.0 & Stomach & 66.8 & 6.3 & $\begin{array}{l}\text { Mouth and } \\
\text { oropharynx }\end{array}$ & 18.6 & 6.4 \\
\hline Prostate & 19.2 & 6.5 & $\begin{array}{l}\text { Lymphomas and } \\
\text { multiple myeloma }\end{array}$ & 55.0 & 5.2 & $\begin{array}{l}\text { Lymphomas and } \\
\text { multiple myeloma }\end{array}$ & 15.8 & 5.4 \\
\hline $\begin{array}{l}\text { Lymphomas and } \\
\text { multiple myeloma }\end{array}$ & 18.8 & 6.4 & Pancreas & 53.6 & 5.0 & Cervix uteri & 15.4 & 5.3 \\
\hline Stomach & 17.0 & 5.7 & Liver & 38.6 & 3.6 & Leukaemia & 14.7 & 5.1 \\
\hline Colon and rectum & 14.1 & 4.8 & Bladder & 37.6 & 3.5 & Stomach & 12.5 & 4.3 \\
\hline $\begin{array}{l}\text { Trachea, bronchus, } \\
\text { and lung }\end{array}$ & 14.0 & 4.7 & Leukaemia & 37.3 & 3.5 & Ovary & 8.7 & 3.0 \\
\hline Leukaemia & 11.8 & 4.0 & Oesophagus & 29.2 & 2.7 & Prostate & 8.1 & 2.8 \\
\hline Ovary & 6.7 & 2.3 & Ovary & 25.7 & 2.4 & Pancreas & 6.8 & 2.3 \\
\hline Bladder & 6.3 & 2.1 & $\begin{array}{l}\text { Mouth and } \\
\text { oropharynx }\end{array}$ & 24.9 & 2.3 & Bladder & 5.8 & 2.0 \\
\hline Melanoma of the skin & 5.3 & 1.8 & Corpus uteri & 16.0 & 1.5 & Oesophagus & 5.6 & 1.9 \\
\hline Pancreas & 5.2 & 1.8 & Melanoma of the skin & 15.5 & 1.5 & Corpus uteri & 4.0 & 1.4 \\
\hline Corpus uteri & 1.7 & 0.6 & Cervix uteri & 8.3 & 0.8 & Melanoma of the skin & 1.7 & 0.6 \\
\hline \multicolumn{9}{|l|}{ Males } \\
\hline Liver & 23.5 & 15.2 & $\begin{array}{l}\text { Trachea, bronchus, } \\
\text { and lung }\end{array}$ & 158.0 & 26.6 & $\begin{array}{l}\text { Trachea, bronchus, } \\
\text { and lung }\end{array}$ & 40.8 & 26.4 \\
\hline Prostate & 19.2 & 12.5 & Colon and rectum & 72.1 & 12.1 & Liver & 25.9 & 16.7 \\
\hline $\begin{array}{l}\text { Mouth and } \\
\text { oropharynx }\end{array}$ & 14.3 & 9.3 & Prostate & 70.2 & 11.8 & Colon and rectum & 13.9 & 9.0 \\
\hline Oesophagus & 13.8 & 8.9 & Stomach & 38.9 & 6.5 & $\begin{array}{l}\text { Mouth and } \\
\text { oropharynx }\end{array}$ & 13.1 & 8.4 \\
\hline $\begin{array}{l}\text { Lymphomas and } \\
\text { multiple myeloma }\end{array}$ & 13.7 & 8.9 & $\begin{array}{l}\text { Lymphomas and } \\
\text { multiple myeloma }\end{array}$ & 27.6 & 4.6 & $\begin{array}{l}\text { Lymphomas and } \\
\text { multiple myeloma }\end{array}$ & 9.6 & 6.2 \\
\hline Stomach & 9.8 & 6.3 & Bladder & 27.1 & 4.6 & Prostate & 8.1 & 5.3 \\
\hline $\begin{array}{l}\text { Trachea, bronchus, } \\
\text { and lung }\end{array}$ & 9.4 & 6.1 & Pancreas & 26.7 & 4.5 & Leukaemia & 7.6 & 4.9 \\
\hline Colon and rectum & 8.8 & 5.7 & Liver & 25.3 & 4.3 & Stomach & 7.1 & 4.6 \\
\hline Leukaemia & 6.9 & 4.5 & Oesophagus & 21.4 & 3.6 & Bladder & 4.4 & 2.8 \\
\hline Bladder & 4.3 & 2.8 & Leukaemia & 20.0 & 3.4 & Pancreas & 3.5 & 2.3 \\
\hline Pancreas & 2.7 & 1.8 & $\begin{array}{l}\text { Mouth and } \\
\text { oropharynx }\end{array}$ & 19.2 & 3.2 & Oesophagus & 3.3 & 2.1 \\
\hline Melanoma of the skin & 2.2 & 1.5 & Melanoma of the skin & 8.3 & 1.4 & Melanoma of the skin & 0.7 & 0.5 \\
\hline \multicolumn{9}{|l|}{ Females } \\
\hline Cervix uteri & 36.8 & 26.0 & Breast & 91.8 & 19.6 & Breast & 29.0 & 21.2 \\
\hline Breast & 23.0 & 16.3 & Colon and rectum & 69.7 & 14.9 & Cervix uteri & 15.4 & 11.2 \\
\hline Liver & 9.8 & 6.9 & $\begin{array}{l}\text { Trachea, bronchus, } \\
\text { and lung }\end{array}$ & 49.2 & 10.5 & Colon and rectum & 13.6 & 10.0 \\
\hline Stomach & 7.2 & 5.1 & Stomach & 27.9 & 6.0 & Liver & 10.6 & 7.8 \\
\hline $\begin{array}{l}\text { Mouth and } \\
\text { oropharynx }\end{array}$ & 7.1 & 5.0 & $\begin{array}{l}\text { Lymphomas and } \\
\text { multiple myeloma }\end{array}$ & 27.4 & 5.9 & Ovary & 8.7 & 6.3 \\
\hline
\end{tabular}


Table 2: Ranking of selected regional cancer deaths by site, 2000 (Continued)

\begin{tabular}{|c|c|c|c|c|c|c|c|c|}
\hline Oesophagus & 6.8 & 4.8 & Pancreas & 26.9 & 5.8 & $\begin{array}{l}\text { Trachea, bronchus, } \\
\text { and lung }\end{array}$ & 7.5 & 5.5 \\
\hline Ovary & 6.7 & 4.8 & Ovary & 25.7 & 5.5 & Leukaemia & 7.2 & 5.2 \\
\hline Colon and rectum & 5.3 & 3.7 & Leukaemia & 17.2 & 3.7 & $\begin{array}{l}\text { Lymphomas and } \\
\text { multiple myeloma }\end{array}$ & 6.1 & 4.5 \\
\hline $\begin{array}{l}\text { Lymphomas and } \\
\text { multiple myeloma }\end{array}$ & 5.2 & 3.7 & Corpus uteri & 16.0 & 3.4 & $\begin{array}{l}\text { Mouth and } \\
\text { oropharynx }\end{array}$ & 5.5 & 4.0 \\
\hline Leukaemia & 4.9 & 3.5 & Liver & 13.3 & 2.8 & Stomach & 5.5 & 4.0 \\
\hline $\begin{array}{l}\text { Trachea, bronchus, } \\
\text { and lung }\end{array}$ & 4.5 & 3.2 & Bladder & 10.5 & 2.2 & Corpus uteri & 4.0 & 2.9 \\
\hline Melanoma of the skin & 3.0 & 2.1 & Cervix uteri & 8.3 & 1.8 & Pancreas & 3.3 & 2.4 \\
\hline Pancreas & 2.5 & 1.8 & Oesophagus & 7.8 & 1.7 & Oesophagus & 2.3 & 1.7 \\
\hline Bladder & 2.0 & 1.4 & Melanoma of the skin & 7.2 & 1.5 & Bladder & 1.4 & 1.0 \\
\hline Corpus uteri & 1.7 & 1.2 & $\begin{array}{l}\text { Mouth and } \\
\text { oropharynx }\end{array}$ & 5.7 & 1.2 & Melanoma of the skin & 1.0 & 0.7 \\
\hline
\end{tabular}

Table 3: Ranking of the global cancer incidence by site, 2000

\begin{tabular}{|c|c|c|}
\hline Site & Number of new cases (000s) & Proportion of total (\%) \\
\hline \multicolumn{3}{|l|}{ Both sexes } \\
\hline Trachea, bronchus and lung & $1,305.6$ & 12.7 \\
\hline Colon and rectum & $1,045.0$ & 10.2 \\
\hline Breast & $1,031.9$ & 10.0 \\
\hline Stomach & 973.1 & 9.5 \\
\hline Liver & 626.4 & 6.1 \\
\hline Prostate & 550.2 & 5.3 \\
\hline Cervix uteri & 487.5 & 4.7 \\
\hline Lymphomas and multiple myeloma & 481.1 & 4.7 \\
\hline Oesophagus & 452.7 & 4.4 \\
\hline Mouth and oropharynx & 420.7 & 4.1 \\
\hline Bladder & 382.9 & 3.7 \\
\hline Leukaemia & 364.7 & 3.5 \\
\hline Corpus uteri & 326.3 & 3.2 \\
\hline Pancreas & 226.2 & 2.2 \\
\hline Ovary & 221.3 & 2.2 \\
\hline Melanoma of the skin & 206.7 & 2.0 \\
\hline \multicolumn{3}{|l|}{ Males } \\
\hline Trachea, bronchus and lung & 939.9 & 18.2 \\
\hline Stomach & 589.3 & 11.4 \\
\hline Prostate & 550.2 & 10.6 \\
\hline Colon and rectum & 527.8 & 10.2 \\
\hline Liver & 422.0 & 8.2 \\
\hline Oesophagus & 286.5 & 5.5 \\
\hline Bladder & 277.2 & 5.4 \\
\hline Mouth and oropharynx & 267.1 & 5.2 \\
\hline Lymphomas and multiple myeloma & 238.5 & 4.6 \\
\hline Leukaemia & 195.2 & 3.8 \\
\hline Pancreas & 119.3 & 2.3 \\
\hline Melanoma of the skin & 118.2 & 2.3 \\
\hline \multicolumn{3}{|l|}{ Females } \\
\hline Breast & $1,031.9$ & 20.1 \\
\hline Colon and rectum & 517.3 & 10.1 \\
\hline Cervix uteri & 487.5 & 9.5 \\
\hline Stomach & 383.9 & 7.5 \\
\hline Trachea, bronchus and lung & 365.7 & 7.1 \\
\hline
\end{tabular}


Table 3: Ranking of the global cancer incidence by site, 2000 (Continued)

\begin{tabular}{lcc}
\hline Corpus uteri & 326.3 & 6.4 \\
Lymphomas and multiple myeloma & 242.6 & 4.7 \\
Ovary & 221.3 & 4.3 \\
Liver & 204.4 & 4.0 \\
Leukaemia & 169.5 & 3.3 \\
Oesophagus & 166.2 & 3.2 \\
Mouth and oropharynx & 153.6 & 3.0 \\
Pancreas & 106.9 & 2.1 \\
Bladder & 105.7 & 2.1 \\
Melanoma of the skin & 88.5 & 1.7 \\
\hline
\end{tabular}

Table 4: Ranking of the selected regional cancer incidence by site, 2000

\begin{tabular}{|c|c|c|c|c|c|c|c|c|}
\hline \multicolumn{9}{|c|}{ WHO sub region } \\
\hline \multicolumn{3}{|c|}{ AfrE (high child and adult mortality) } & \multicolumn{3}{|c|}{ EurA (very low child and adult mortality) } & \multicolumn{3}{|c|}{ SearB (low child and adult mortality) } \\
\hline Site & $\begin{array}{l}\text { Number } \\
\text { of new } \\
\text { cases } \\
(000 s)\end{array}$ & $\begin{array}{l}\text { Proportion } \\
\text { of total (\%) }\end{array}$ & Site & $\begin{array}{l}\text { Number } \\
\text { of new } \\
\text { cases } \\
(000 s)\end{array}$ & $\begin{array}{l}\text { Proportion } \\
\text { of total (\%) }\end{array}$ & Site & $\begin{array}{l}\text { Number } \\
\text { of new } \\
\text { cases } \\
(000 \mathrm{~s})\end{array}$ & $\begin{array}{l}\text { Proportion } \\
\text { of total (\%) }\end{array}$ \\
\hline \multicolumn{9}{|l|}{ Both sexes } \\
\hline Cervix uteri & 67.9 & 16.4 & Colon and rectum & 264.7 & 15.4 & Breast & 53.9 & 12.3 \\
\hline Breast & 39.8 & 9.6 & $\begin{array}{l}\text { Trachea, bronchus } \\
\text { and lung }\end{array}$ & 227.3 & 13.2 & $\begin{array}{l}\text { Trachea, bronchus } \\
\text { and lung }\end{array}$ & 51.4 & 11.7 \\
\hline Liver & 34.7 & 8.3 & Breast & 221.8 & 12.9 & Colon and rectum & 44.3 & 10.1 \\
\hline Prostate & 28.2 & 6.8 & Prostate & 139.1 & 8.1 & Liver & 40.3 & 9.2 \\
\hline $\begin{array}{l}\text { Mouth and } \\
\text { oropharynx }\end{array}$ & 27.9 & 6.7 & Bladder & 86.5 & 5.0 & Cervix uteri & 32.8 & 7.5 \\
\hline $\begin{array}{l}\text { Lymphomas and } \\
\text { multiple myeloma }\end{array}$ & 26.6 & 6.4 & $\begin{array}{l}\text { Lymphomas and } \\
\text { multiple myeloma }\end{array}$ & 80.2 & 4.7 & $\begin{array}{l}\text { Mouth and } \\
\text { oropharynx }\end{array}$ & 25.4 & 5.8 \\
\hline Oesophagus & 21.2 & 5.1 & Stomach & 79.6 & 4.6 & $\begin{array}{l}\text { Lymphomas and } \\
\text { multiple myeloma }\end{array}$ & 22.9 & 5.2 \\
\hline Colon and rectum & 20.3 & 4.9 & Corpus uteri & 68.3 & 4.0 & Leukaemia & 21.5 & 4.9 \\
\hline Stomach & 19.4 & 4.7 & Pancreas & 55.5 & 3.2 & Corpus uteri & 19.9 & 4.5 \\
\hline Leukaemia & 16.2 & 3.9 & Melanoma of the skin & 49.1 & 2.8 & Ovary & 18.1 & 4.1 \\
\hline $\begin{array}{l}\text { Trachea, bronchus } \\
\text { and lung }\end{array}$ & 14.8 & 3.6 & Leukaemia & 48.6 & 2.8 & Stomach & 15.3 & 3.5 \\
\hline Ovary & 12.2 & 2.9 & Liver & 40.1 & 2.3 & Prostate & 13.4 & 3.1 \\
\hline Bladder & 10.7 & 2.6 & Ovary & 39.4 & 2.3 & Bladder & 10.1 & 2.3 \\
\hline Melanoma of the skin & 7.7 & 1.8 & $\begin{array}{l}\text { Mouth and } \\
\text { oropharynx }\end{array}$ & 38.6 & 2.2 & Pancreas & 7.2 & 1.7 \\
\hline Corpus uteri & 6.0 & $\mathrm{I} .4$ & Oesophagus & 31.2 & 1.8 & Oesophagus & 6.2 & 1.4 \\
\hline Pancreas & 5.4 & 1.3 & Cervix uteri & 18.8 & 1.1 & Melanoma of the skin & 3.1 & 0.7 \\
\hline Male & & & Male & & & Male & & \\
\hline Prostate & 28.2 & 14.8 & $\begin{array}{l}\text { Trachea, bronchus } \\
\text { and lung }\end{array}$ & 172.0 & 19.6 & $\begin{array}{l}\text { Trachea, bronchus } \\
\text { and lung }\end{array}$ & 43.1 & 22.0 \\
\hline Liver & 23.6 & 12.4 & Prostate & 139.1 & 15.9 & Liver & 28.6 & 14.6 \\
\hline $\begin{array}{l}\text { Lymphomas and } \\
\text { multiple myeloma }\end{array}$ & 18.6 & 9.8 & Colon and rectum & 133.8 & 15.3 & Colon and rectum & 21.4 & 11.0 \\
\hline $\begin{array}{l}\text { Mouth and } \\
\text { oropharynx }\end{array}$ & 16.5 & 8.7 & Bladder & 65.1 & 7.4 & $\begin{array}{l}\text { Mouth and } \\
\text { oropharynx }\end{array}$ & 16.0 & 8.2 \\
\hline Oesophagus & 14.1 & 7.4 & Stomach & 45.6 & 5.2 & Prostate & 13.4 & 6.9 \\
\hline Colon and rectum & 12.0 & 6.3 & $\begin{array}{l}\text { Lymphomas and } \\
\text { multiple myeloma }\end{array}$ & 39.3 & 4.5 & $\begin{array}{l}\text { Lymphomas and } \\
\text { multiple myeloma }\end{array}$ & 13.3 & 6.8 \\
\hline Stomach & 10.9 & 5.8 & $\begin{array}{l}\text { Mouth and } \\
\text { oropharynx }\end{array}$ & 28.7 & 3.3 & Leukaemia & 10.7 & 5.5 \\
\hline
\end{tabular}


Table 4: Ranking of the selected regional cancer incidence by site, 2000 (Continued)

\begin{tabular}{|c|c|c|c|c|c|c|c|c|}
\hline $\begin{array}{l}\text { Trachea, bronchus } \\
\text { and lung }\end{array}$ & 9.8 & 5.2 & Pancreas & 27.4 & 3.1 & Stomach & 8.6 & 4.4 \\
\hline Leukaemia & 9.2 & 4.9 & Liver & 26.1 & 3.0 & Bladder & 7.7 & 3.9 \\
\hline Bladder & 7.3 & 3.8 & Leukaemia & 25.6 & 2.9 & Oesophagus & 3.8 & 1.9 \\
\hline Melanoma of the skin & 3.4 & 1.8 & Oesophagus & 22.8 & 2.6 & Pancreas & 3.7 & 1.9 \\
\hline Pancreas & 2.8 & 1.5 & Melanoma of the skin & 22.6 & 2.6 & Melanoma of the skin & 1.2 & 0.6 \\
\hline Female & & & Female & & & Female & & \\
\hline Cervix uteri & 67.9 & 30.2 & Breast & 221.8 & 26.2 & Breast & 53.9 & 22.3 \\
\hline Breast & 39.8 & 17.7 & Colon and rectum & 131.0 & 15.4 & Cervix uteri & 32.8 & 13.6 \\
\hline Ovary & 12.2 & 5.4 & Corpus uteri & 68.3 & 8.1 & Colon and rectum & 22.8 & 9.4 \\
\hline $\begin{array}{l}\text { Mouth and } \\
\text { oropharynx }\end{array}$ & I I.4 & 5.1 & $\begin{array}{l}\text { Trachea, bronchus } \\
\text { and lung }\end{array}$ & 55.3 & 6.5 & Corpus uteri & 19.9 & 8.2 \\
\hline Liver & II. I & 4.9 & $\begin{array}{l}\text { Lymphomas and } \\
\text { multiple myeloma }\end{array}$ & 40.9 & 4.8 & Ovary & 18.1 & 7.5 \\
\hline Stomach & 8.4 & 3.7 & Ovary & 39.4 & 4.6 & Liver & | I.7 & 4.8 \\
\hline Colon and rectum & 8.2 & 3.7 & Stomach & 33.9 & 4.0 & Leukaemia & 10.8 & 4.5 \\
\hline $\begin{array}{l}\text { Lymphomas and } \\
\text { multiple myeloma }\end{array}$ & 8.0 & 3.6 & Pancreas & 28.1 & 3.3 & $\begin{array}{l}\text { Lymphomas and } \\
\text { multiple myeloma }\end{array}$ & 9.6 & 4.0 \\
\hline Oesophagus & 7.2 & 3.2 & Melanoma of the skin & 26.5 & 3.1 & $\begin{array}{l}\text { Mouth and } \\
\text { oropharynx }\end{array}$ & 9.4 & 3.9 \\
\hline Leukaemia & 7.0 & 3.1 & Leukaemia & 23.0 & 2.7 & $\begin{array}{l}\text { Trachea, bronchus } \\
\text { and lung }\end{array}$ & 8.3 & 3.4 \\
\hline Corpus uteri & 6.0 & 2.7 & Bladder & 21.4 & 2.5 & Stomach & 6.7 & 2.8 \\
\hline $\begin{array}{l}\text { Trachea, bronchus } \\
\text { and lung }\end{array}$ & 5.0 & 2.2 & Cervix uteri & 18.8 & 2.2 & Pancreas & 3.6 & 1.5 \\
\hline Melanoma of the skin & 4.3 & 1.9 & Liver & 14.0 & 1.7 & Oesophagus & 2.5 & 1.0 \\
\hline Bladder & 3.4 & 1.5 & $\begin{array}{l}\text { Mouth and } \\
\text { oropharynx }\end{array}$ & 9.9 & 1.2 & Bladder & 2.4 & 1.0 \\
\hline Pancreas & 2.6 & 1.2 & Oesophagus & 8.4 & 1.0 & Melanoma of the skin & 1.9 & 0.8 \\
\hline
\end{tabular}

Publish with Bio Med Central and every scientist can read your work free of charge

"BioMed Central will be the most significant development for disseminating the results of biomedical research in our lifetime. "

Sir Paul Nurse, Cancer Research UK

Your research papers will be:

- available free of charge to the entire biomedical community

- peer reviewed and published immediately upon acceptance

- cited in PubMed and archived on PubMed Central

- yours - you keep the copyright 